\title{
Edición universitaria y políticas editoriales como objeto de análisis: Prácticas de lectura y políticas públicas Proyecto de Investigación \\ $\mathrm{N}^{\circ} 6.2$ \\ Equipo de Investigación ${ }^{(1)}$ \\ Ivana Mihal \\ Daniela Szpilbarg
}

por Facultad de Diseño y Comunicación, Universidad de Palermo (ARG)

por Instituto de Desarrollo Económico y Social, IDES-CONICET (ARG)

Resumen: El Proyecto de Investigación 6.2 indaga en la edición universitaria desde una pluralidad de puntos de vista e interpretaciones. Se enfoca en la identificación, revisión y análisis de aspectos que ayudan a comprender y explicar el panorama actual de las editoriales cuya existencia y desarrollo tiene lugar en el ámbito de las universidades, y desde allí reflexionar sobre algunos desafíos para el futuro. Gran parte de la investigación científica y de otros tipos de producciones editoriales en años recientes ha sido publicada en estos espacios. Su creciente internacionalización y profesionalización ha permitido ir construyendo su consolidación en el sector editorial de la mano del crecimiento y marco de las universidades. El estudio de ésta temática aún presenta un área de vacancia en los estudios sobre políticas editoriales en instituciones de educación superior.

Palabras clave: Edición Universitaria - Políticas Editoriales - Libros Universitarios - Digitalización - Profesionalización.

[Resúmenes en inglés y portugués en la página 176]

${ }^{(1)}$ Los CVs del Equipo de Investigación pueden consultarse en el Capítulo Directores de Líneas y Coordinadores de Proyectos de esta misma Edición.

\section{Acerca del Proyecto 6.2}

Edición universitaria y políticas editoriales como objeto de análisis:

Prácticas de lectura y políticas públicas

El Proyecto 6.2 Edición universitaria y políticas editoriales como objeto de análisis: Prácticas de lectura y políticas públicas, reúne diversas perspectivas, en un estudio inter- 
disciplinario en el marco de las políticas públicas, siendo que el mundo digital ha tenido un significativo desarrollo en los últimos tiempos, que incide sobre casi todas las actividades del ser humano, por tanto también, en las prácticas de lectura. La industria del libro y las nuevas y/o renovadas configuraciones en los modos de leer ameritan un acercamiento esta particular problemática, centrando el campo específico de los estudios en la cultura y la creatividad, para que sea posible identificar e indagar los alcances y desafíos que se plantean.

Se acordó entre la Universidad de Palermo (Argentina) y el Instituto de Desarrollo Económico y Social, IDES-CONICET (Argentina), avanzar en una investigación en la que participan investigadores, provenientes de distintas disciplinas, como comunicación, diseño, historia, antropología, sociología, ciencias de la educación, entre otras carreras afines, con la coordinación compartida de Ivana Mihal (UP) y Daniela Szpilbarg (IDES-CONICET), con el fin de abordar las transformaciones socioculturales en las prácticas, en las concepciones, y en las políticas que se entretejen a la hora de pensar la problemática, e Impactar en la formación de los Diseñadores Gráficos, puntualmente a través de los contenidos del Diseño Editorial y la Lectura digital.

Las reflexiones y los resultados obtenidos en el Proyecto 6.2 Edición universitaria y políticas editoriales como objeto de análisis son continuación del Proyecto 6.1 Lectura Digital: Incidencia de la práctica digital en los hábitos de lectura, y se continúan desarrollando en el Proyecto 6.3 Producción, Circulación y Comunicación del conocimiento en Instituciones de Educación Superior: Políticas Editoriales, en el marco de la misma Línea de Investigación y bajo la misma Directora Ivana Mihal.

Sus principales objetivos son:

- Estudiar los ámbitos concretos de abordaje del diseño, la comunicación y la edición de libros digitales en relación con las problemáticas de lectura y escritura, tanto como la producción y circulación de libros en distintos soportes;

- Analizar la incidencia del mundo digital en las prácticas de lectura y en las producciones específicas de la industria editorial digitalizada;

- Explorar los entrecruzamientos de libros impresos y lo digital

- Estudiar, desde distintas perspectivas, la incidencia de lo digital en el diálogo libros y lecturas con el diseño y la comunicación, junto con los procesos que involucran estos temas;

- Ahondar en los consumos culturales de la sociedad contemporánea, mediante el análisis del sector editorial de libros y las distintas maneras de leer en la actualidad - Impactar en el Diseño Editorial, con la actualización de los conocimientos transitados en la carrera de Diseño Gráfico en relación con la lectura digital.

\section{Acerca de la Línea 6}

Convergencia pedagógica digital: Miradas interdisciplinarias dirigida por Ivana Mihal se desarrolla de manera ininterrumpida desde 2015 en la Facultad de Diseño y Comunicación (UP, Argentina), e incluye hasta el momento tres proyectos finalizados el $6.1 \mathrm{Lec}-$ 
tura Digital: Incidencia de la práctica digital en los hábitos de lectura coordinado por Ivana Mihal, el 6.2 Edición universitaria y políticas editoriales como objeto de análisis coordinado por Ivana Mihal (UP) y Daniela Szpilbarg (Instituto de Desarrollo Económico y Social, IDES-CONICET, Argentina), y el 6.3 Producción, Circulación y Comunicación del conocimiento en Instituciones de Educación Superior: Políticas Editoriales, coordinado por Ivana Mihal (Universidad de Palermo, y Laboratorio de Investigación en Ciencias Humanas de la UNSAM-CONICET, Argentina), Ana Elisa Ribeiro (Centro Federal de Educacao Tecnológica de Minas Gerais-CEFET-MG, Brasil) y Daniela Szpilbarg (Instituto de Desarrollo Económico y Social, IDES-CONICET, Argentina).

\section{Mapa de Áreas y Proyectos}

El Proyecto 6.2 se vincula con todas las carreras de grado y posgrado correspondientes a la Facultad de Diseño y Comunicación dado que impacta en la incorporación de la investigación en la lógica de la docencia universitaria, y específicamente de manera fundamental con la carrera de Diseño gráfico y Comunicación digital. Además los proyectos de la Línea de Investigación están vinculados también con los de la Línea de Investigación N9 Enseñar Disciplinas Proyectuales.

\section{Productos y Resultados}

\section{a)- Publicaciones}

Cuaderno del Centro de Estudios de Diseño y Comunicación N85. (2020) Edición universitaria y políticas editoriales como objeto de análisis. Facultad de Diseño y Comunicación Año XIX, Noviembre 2020, Buenos Aires, Argentina. ISSN: 1668-0227. Esta publicación documenta y comunica los resultados alcanzados en el Proyecto 6.2 Edición universitaria y políticas editoriales como objeto de análisis (finalizado), y a continuación se detallan los autores $y$ artículos contenidos en ella:

Ivana Mihal, Daniela Szpilbarg (2020) Prólogo: Edición universitaria y políticas editoriales como objeto de análisis (Pp. 11 a 19)

Carlos Zelarayán (2020) Encrucijadas de la edición universitaria (Pp. 21 a 34)

Alejandro Dujovne (2020) Gutenberg atiende en Buenos Aires. La edición universitaria ante la concentración geográfica del mercado editorial argentino (Pp. 35 a 47)

Ivana Mihal (2020) La edición universitaria argentina a la luz de la Feria del Libro de Guadalajara: acerca de la internacionalización y digitalización (Pp. 49 a 69)

Emanuel Molina (2020) El armado de un catálogo en una editorial universitaria. El caso de la Editorial Universitaria Villa María (Pp. 71 a 84)

Ana Verdelli (2020) Las editoriales universitarias de cara a los procesos de internacionalización de la educación superior: El caso de las políticas editoriales de EDUNTR (Pp. 85 a 94) 
Guido Olivares (2020) Presencia de las Editoriales Universitarias en las convocatorias del Fondo del Libro, Chile. 2013-2018 (Pp. 95 a 106)

Juan Felipe Córdoba Restrepo (2020) Editar en la universidad, una construcción permanente (Pp. 107 a 118)

Daniela Szpilbarg (2020) Políticas editoriales y digitalización. El caso de EUDEBA y el lector digital "Boris" (Pp.119 a 134)

Jorge M. Gorostiaga (2020) Digitalización en las revistas académicas de educación en Argentina (Pp. 135 a 150)

Micaela Persson (2020) La Internacionalización de la Educación Superior a través de las revistas científicas digitales en América Latina (Pp. 151 a 164)

Ana Slimovich, Ezequiel Saferstein (2020) Análisis sobre los modos digitales de difusión de las grandes editoriales en Argentina: libros de "coyuntura política" en las redes sociales (Pp. 165 a 188)

Actas de Diseño No30 (2019) Semana Internacional del Diseño en Palermo. Facultad de Diseño y Comunicación, Universidad de Palermo. En esta publicación se documentan las ponencias de la Comisión Convergencia pedagógico digital: Proyecto 6.2 Edición universitaria y políticas editoriales como objeto de análisis correspondientes a la presentación del Cuaderno 85 durante el IV Coloquio de Investigación y Desarrollo en Diseño (Pp.43-44).

\section{b)- Congresos / Coloquios / Plenarios}

$3^{\circ}$ Plenario de Directores de Investigación DC. 26, 27 y 29 de agosto de 2019. En este tercer plenario los Directores de las Líneas y Proyectos de Investigación presentaron a sus pares y al conjunto del Programa de Investigación de la Facultad de Diseño y Comunicación, los resultados obtenidos y/o en proceso (publicaciones y acuerdos), junto con los avances de Proyectos. La Directora de la Línea de Investigación Nº6 presentó a sus pares, los resultados del Proyecto 6.2 Edición universitaria y políticas editoriales como objeto de análisis.

IV Coloquio de Investigación y Desarrollo en Diseño Latino. Universidad de Palermo, 29 de julio de 2019. X Congreso Latinoamericano de Enseñanza del Diseño. Semana Internacional del Diseño en Palermo.

En la comisión coordinada por Ivana Mihal y Daniela Szpilbarg se expusieron las conclusiones del proyecto 6.2 Edición universitaria y políticas editoriales como objeto de análisis, condensadas en el Cuaderno 85 del mismo nombre. El debate se centró sobre la exploración de las políticas editoriales en instituciones de educación superior, las transformaciones y/o reelaboraciones que vienen desarrollándose con la producción y comunicación del conocimiento; con la lectura digital; como con problemáticas específicas que atraviesan las universidades y las editoriales universitarias en dicho campo.

Expusieron: Ivana Mihal, Daniela Szpilbarg, Carlos Zelarayán, Alejandro Dujovne, Emanuel Molina, Guido Olivares, Juan Felipe Córdoba Restrepo, Micaela Persson y Ana Slimovich. 
A continuación se detallan las ponencias presentadas en esta Comisión coordinada por Ivana Mihal y Daniela Szpilbarg:

Ivana Mihal y Daniela Szpilbarg (Argentina) - Coordinadora de la Comisión

Presentación Cuadernos "Edición universitaria y políticas editoriales como objeto de análisis"

Ivana Mihal (Argentina)

La edición universitaria argentina a la luz de la Feria del Libro de Guadalajara: acerca de la internacionalización y digitalización

Daniela Szpilbarg (Argentina)

Políticas editoriales y digitalización. El caso de EUDEBA y el lector digital "Boris"

Carlos Zelarayán (Argentina)

Encrucijadas de la edición universitaria

Alejandro Dujovne (Argentina)

Gutenberg atiende en Buenos Aires. La edición universitaria ante la concentración geográfica del mercado editorial argentino

Emanuel Molina (Argentina)

El armado de un catálogo en una editorial universitaria

Guido Olivares (Chile)

Presencia de las editoriales regionales en las convocatorias del Fondo del Libro, Chile Juan Felipe Córdoba Restrepo (Argentina)

Editar en la universidad, una construcción permanente

Micaela Persson (Argentina)

La Internacionalización de la Educación Superior a través de las revistas científicas digitales en América Latina

Ana Slimovich (Argentina)

Análisis sobre los modos digitales de difusión de las grandes editoriales en Argentina: libros de "coyuntura política"

$2^{\circ}$ Plenario de Directores de Investigación DC, 27 y 4 de septiembre - 2018. Es este segundo plenario los Directores de las Líneas y Proyectos de Investigación presentaron a sus pares y al conjunto del Programa de Investigación de la Facultad de Diseño y Comunicación, los resultados obtenidos y/o en proceso (publicaciones y acuerdos), junto con los avances de Proyectos. La Directora de la Línea de Investigación №6 presentó a sus pares, los resultados del Proyecto 6.1 Lectura Digital, y anticiparon los comienzos del Proyecto 6.2 Edición universitaria y políticas editoriales como objeto de análisis.

III Coloquio de Investigación y Desarrollo en Diseño Latino. Universidad de Palermo, 30 de julio de 2018. IX Congreso Latinoamericano de Enseñanza del Diseño. Semana Internacional del Diseño en Palermo.

En la comisión Convergencia pedagógica digital: miradas Interdisciplinarias, se presentaron las reflexiones y conclusiones del proyecto 6.1 Lectura Digital (Finalizado) expusieron: Mariana Landau, Ana Ligia Medeiros, Gilda Olinto, María del Carmen Rosas Franco, Mónica Pini, Daniela Szpilbarg e Ivana Mihal. 
A continuación se detallan las ponencias presentadas en esta Comisión coordinada por la Directora del proyecto Ivana Mihal:

\title{
Landau, Mariana
}

Los discursos sobre tecnologías y educación en la esfera pública

Medeiros Ana Ligia, Olinto Gilda

O impacto da tecnologia de informação e comunicação nas bibliotecas públicas: envolvimento comunitário, criatividade e inovação

Rosas Franco, María del Carmen

Nuevos soportes, nuevos modos de leer. La narrativa en la literatura infantil y juvenil digital

Pini Mónica

Políticas de alfabetización digital. Educación e inclusión

Mihal Ivana

Lectura digital y lectura transmedia: pensando el lugar del libro y de las editoriales Spizpilbarg Daniela

La profesionalización de las editoriales universitarias en Argentina (2010-2015). Prácticas editoriales emergentes y digitalización

\section{c)- Formación de Posgrado e Impacto curricular}

La Directora Ivana Mihal es Docente de Posgrado en Diseño de la Facultad de Diseño y Comunicación en Maestría en Gestión del Diseño y Doctorado en Diseño y al igual que Daniela Spilzbarg en su Institución (IDES-CONICET), incorporan los contenidos de su investigación a sus asignaturas en posgrado.

\begin{abstract}
Research Project 6.2 investigates university publishing from a plurality of points of view and interpretations. It focuses on the identification, review and analysis of aspects that help to understand and explain the current panorama of publishers whose existence and development takes place in the field of universities, and from there reflect on some challenges for the future. Much of the scientific research and other types of editorial productions in recent years have been published in these spaces. Its increasing internationalization and professionalization has allowed it to build its consolidation in the publishing sector hand in hand with the growth and framework of the universities. The study of this topic still presents a vacancy area in studies on editorial policies in higher education institutions.
\end{abstract}

Keywords: University Publishing - Editorial Policies - University Books - Digitization Professionalization. 
Resumo: O Projeto de Pesquisa 6.2 investiga a publicação universitária de uma pluralidade de pontos de vista e interpretações. Tem como foco a identificação, revisão e análise de aspectos que ajudem a compreender e explicar o panorama atual das editoras cuja existência e desenvolvimento se dão no campo das universidades, e a partir daí refletir sobre alguns desafios para o futuro. Muitas das pesquisas científicas e outros tipos de produções editoriais dos últimos anos foram publicadas nesses espaços. A sua crescente internacionalização e profissionalização tem permitido construir a sua consolidação no sector editorial a par do crescimento e enquadramento das universidades. O estudo deste tema apresenta ainda uma vaga em estudos sobre políticas editoriais em instituições de ensino superior.

Palavras chave: Publicação Universitária - Política Editorial - Livros Universitários - Digitalização - Profissionalização.

[Las traducciones de los resúmenes fueron realizadas a través de traductor automático] 